\title{
Prolonged Intermittent Renal Replacement Therapy for Acute Kidney Injury in COVID-19 Patients with Acute Respiratory Distress Syndrome
}

\author{
Juan C. Ramirez-Sandovala Jorge E. Gaytan-Arocha ${ }^{a} \quad$ Pedro Xolalpa-Chávez ${ }^{a}$ \\ Juan M. Mejia-Vilet ${ }^{a} \quad$ Mauricio Arvizu-Hernandez ${ }^{a} \quad$ Eduardo Rivero-Sigarroab $^{b}$ \\ Carlos Torruco-Sotelo $^{b}$ Ricardo Correa-Rotter ${ }^{\mathrm{a}}$ Olynka Vega-Vega ${ }^{\mathrm{a}}$ \\ a Department of Nephrology and Mineral Metabolism, Instituto Nacional de Ciencias Médicas y Nutrición Salvador \\ Zubirán, Mexico City, Mexico; bepartment of Intensive Care, Instituto Nacional de Ciencias Médicas y Nutrición \\ Salvador Zubirán, Mexico City, Mexico
}

\section{Keywords}

Renal failure - SARS-CoV-2 - Acute respiratory distress syndrome · Hybrid therapies · Sustained low-efficiency dialysis

\begin{abstract}
Introduction: Patients with acute respiratory distress syndrome (ARDS) secondary to COVID-19 frequently develop severe acute kidney injury (AKI). Although continuous renal replacement therapy is the standard of care for critically ill patients, prolonged intermittent renal replacement therapy (PIRRT) may be a feasible option. We aimed to describe the tolerability and security of PIRRT treatments in COVID-19 patients with ARDS who required mechanical ventilation and developed severe AKI. Methods: We prospectively analyzed patients who underwent PIRRT treatments at a COVID-19 reference hospital in Mexico City. Intradialytic hypotension was defined as a systolic blood pressure decrease of $\geq 20 \mathrm{~mm} \mathrm{Hg}$ or an increase of $100 \%$ in vasopressor dose. Results: We
\end{abstract}

identified $136 \mathrm{AKI}$ cases (60.7\%) in 224 patients admitted to the intensive care unit. Among them, 21 (15\%) underwent PIRRT (130 sessions) due to stage 3 AKI. The median age of the cohort was 49 (range 36-73) years, 17 (81\%) were male, 7 (33\%) had diabetes, and the median time between symptoms onset and PIRRT initiation was 12 (interquartile range [IQR] 7-14) days. The median of PIRRT procedures for each patient was 5 (IQR 4-9) sessions. In 108 (83\%) PIRRT sessions, the total ultrafiltration goal was achieved. In 84 (65\%) PIRRT procedures, there was a median increase in norepinephrine dose of $+0.031 \mathrm{mcg} / \mathrm{kg} / \mathrm{min}$ during PIRRT (IQR 0.00 to +0.07 ). Intradialytic hypotensive events occurred in 56 (43\%) procedures. Fifteen (12\%) PIRRT treatments were discontinued due to severe hypotension. Vasopressor treatment at PIRRT session onset (OR 6.2, 95\% Cl 1.4-28.0, p: 0.02) and a prePIRRT lactate $\geq 3.0 \mathrm{mmol} / \mathrm{L}(\mathrm{OR} 4.63,95 \% \mathrm{Cl} 1.3-12.8, p: 0.003$ ) were independently and significantly associated with the risk of hypotension during PIRRT. During follow-up, 11 patients (52\%) recovered from AKI and respiratory failure and 9 (43\%) died. Several adaptations to our PIRRT protocol dur- karger@karger.com

(C) 2020 S. Karger AG, Basel

www.karger.com/bpu

Karger ${ }^{\prime}=$
Olynka Vega-Vega

Department of Nephrology and Mineral Metabolism

Instituto Nacional de Ciencias Médicas y Nutrición Salvador Zubirán

Vasco de Quiroga 15, Colonia Seccion XVI, Mexico 14080 (Mexico)

olynkavega@ hotmail.com 
ing the COVID-19 outbreak are presented. Conclusions: PIRRT was feasible in the majority of COVID-19 patients with ARDS and severe AKI, despite frequent transitory intradialytic hypotensive episodes. PIRRT may represent an acceptable alternative of renal replacement therapy during the COVID-19 outbreak.

(C) 2020 S. Karger AG, Basel

\section{Introduction}

As the COVID-19 pandemic unfolds in several countries, a high number of patients with severe acute kidney injury (AKI) secondary to COVID-19 pneumonia have become evident. Although continuous renal replacement therapy (CRRT) is the treatment of choice for hemodynamically unstable critically ill patients, its use implies a higher complexity, more extensive training and timeconsuming procedures for nursing staff, the need for continuous anticoagulation, availability of multiple CRRT equipment, and significantly higher costs $[1,2]$. Other treatments, as sequential extracorporeal therapies, are an option for COVID-19 patients with multiple organ failure, as they provide all forms of organ support $[3,4]$ and reduce the level of various inflammatory mediators [5]. Nevertheless, these therapies are not available in many institutions.

Prolonged intermittent renal replacement therapy (PIRRT), also known as sustained low-efficiency dialysis or slow extended daily dialysis, may be an option for critically ill COVID-19 patients given its advantages related to flexible treatment schedules, acceptable hemodynamical tolerability, less nursing time, and lower costs than CRRT [2]. Nevertheless, PIRRT could be inconvenient for COVID-19 patients, who commonly exhibit an exuberant inflammatory response, severe hemodynamic instability, and hypercoagulability. In this article, we describe our experience of 130 PIRRT treatments in $21 \mathrm{CO}-$ VID-19 patients with acute respiratory distress syndrome (ARDS) who required mechanical ventilation and developed severe AKI, most of them with hemodynamic instability. Therefore, we present several adaptations to our PIRRT protocol after the COVID-19 outbreak.

\section{Materials and Methods}

This is a single-center, prospective, observational study performed at the Instituto Nacional de Ciencias Médicas y Nutrición Salvador Zubirán, a tertiary reference hospital designated to treat exclusively patients with COVID-19 in Mexico City since March
16 , 2020. From this date to June 24,2020 , we prospectively analyzed all PIRRT treatments performed in COVID-19 subjects. SARS-CoV-2 infection was confirmed by RT-PCR in all the cases. The Human Research and Ethics Committees of our institution approved our study (reference number NMM-3325-20-20-1). Written informed consent was waived due to the observational nature of the study.

All the included patients had moderate to severe ARDS $\left(\mathrm{PaO}_{2} /\right.$ $\mathrm{FiO}_{2} \leq 200 \mathrm{~mm} \mathrm{Hg}$ ) according to the Berlin ARDS definitions [6] and were receiving mechanical ventilation. The choice between PIRRT and CRRT as the initial renal replacement therapy (RRT) was made at the discretion of the consulting nephrologist who considered, in addition to the clinical characteristics of each patient, the shortage of medical personnel, nursery staff, and RRT supplies during the start of the pandemic. Each patient received $\geq 3$ treatment sessions with high-flux dialyzers (clearance Qb $200 \mathrm{~mL} / \mathrm{min}$, urea 192, effective surface $1.9 \mathrm{~m}^{2}$, DIAPES ${ }^{\circledR}$, Bellco, Italy). All hemodialysis machines used were $4008 \mathrm{~S}\left({ }^{\circledR}\right.$ Fresenius, Germany). All consulting nephrologists prescribed a total fluid removal per hour of $<400 \mathrm{~mL} / \mathrm{h}$ per institutional protocol. We collected data on age, sex, previous comorbidities, symptoms from onset to hospital admission and to PIRRT initiation, laboratory values on PIRRT initiation, treatment (vasoconstrictive agents), and living status.

The outcomes included successful PIRRT according to dialytic and ultrafiltrate goals prescription, events of intradialytic hypotension, system clotting or other adverse events during the PIRRT procedure, mortality, and renal recovery. Intradialytic hypotension was defined as a systolic blood pressure decrease $\geq 20 \mathrm{~mm} \mathrm{Hg}$ or an increase of $100 \%$ in baseline vasopressor dose [7]. Severe intradialytic hypotension was defined as an intradialytic hypotensive episode that required PIRRT interruption. Renal recovery was defined as RRT independence $>14$ days after intensive care unit (ICU) discharge.

We expressed categorical variables as numbers (percentages) and continuous variables as mean \pm standard deviation (SD) or median (interquartile range [IQR]), as appropriate. Comparison between groups was performed with a $t$ test or the Mann-Whitney test for continuous variables and $\chi^{2}$ test or Fisher's test for categorical variables. Variables associated with intradialytic hypotensive episodes with a $p$ value below 0.1 in the univariate analysis were selected for inclusion into a multivariate logistic regression model using forward stepwise selection. Statistical analysis and graphics were performed with SPSS 21.0 software (SPSS Inc., Chicago, IL, USA) and GraphPad Prism 5 (San Diego, CA, USA), respectively. The results were considered statistically significant when $p<0.05$.

\section{Results}

We identified 224 COVID-19 patients admitted to our ICU; 136 (60.7\%) of them developed hospital-acquired AKI, and among them, 39 (29\%) had stage 3 AKI according to KDIGO definitions [8]. In these patients, 21 (15\%) initiated PIRRT. We excluded patients who were treated initially with CRRT instead of PIRRT $(n=3)$. The rest of stage 3 AKI cases were no candidates for RRT given their clinical status (see Fig. 1). 
Fig. 1. Flowchart of the patients included. AKI, acute kidney injury; ICU, intensive care unit; CRRT, continuous renal replacement therapy; RRT, renal replacement therapy; PIRRT, prolonged intermittent renal replacement therapy.

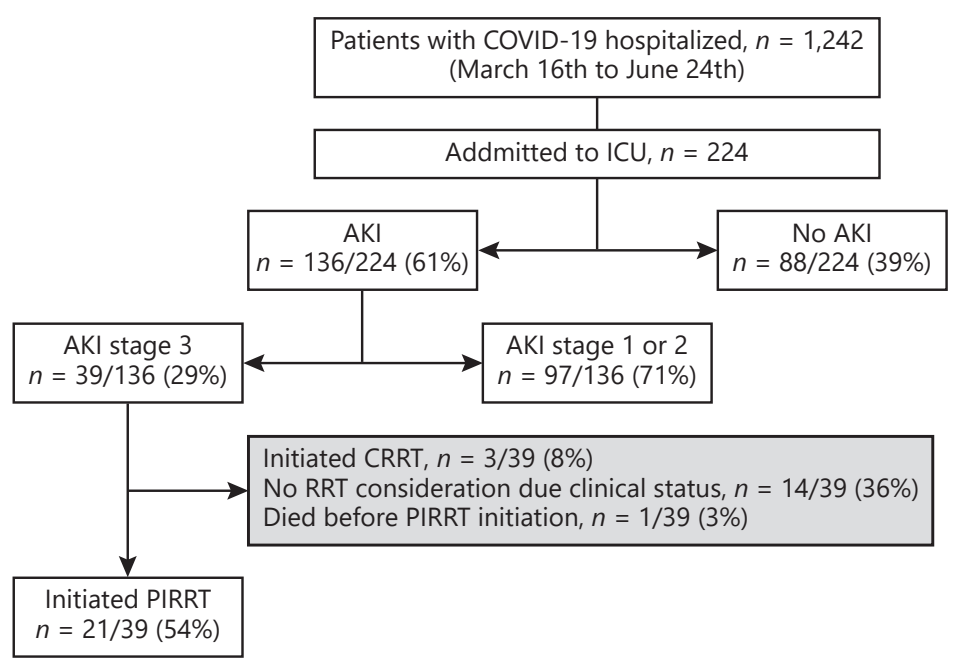

We analyzed 130 PIRRT sessions administered to 21 patients. The median age was 49 (IQR 45-59, range 3673 ) years, $17(81 \%)$ were male, 7 (33\%) had diabetes, and $8(38 \%)$ had a previous diagnosis of systemic hypertension. The median BMI was 31 (IQR 28-36, range 22-51) $\mathrm{kg} / \mathrm{m}^{2}$, median SOFA score was 10 (IQR 9-11) points, and median time between symptoms onset and PIRRT initiation was 12 (IQR 7-14) days. All but 1 patient had fever $\left(\geq 38^{\circ} \mathrm{C}\right)$ within $24 \mathrm{~h}$ before PIRRT initiation. PIRRT was started $72 \mathrm{~h}$ after intubation in all patients, except in 3 who had life-threatening dialysis indications (hyperkalemia and severe mixed acidosis) and who started therapy earlier at their admission. Other clinical characteristics are shown in Table 1.

The median of PIRRT procedures for each patient was 5 (IQR 4-9) (Table 2). In 108 (83\%) PIRRT sessions, the ultrafiltration goal was achieved. The duration of PIRRT was $6-8 \mathrm{~h}$ in 91 treatments (70\%), as planned. In 17 (13\%) sessions, the PIRRT procedure was extended 2-4 additional hours in addition to the original prescription to complete the ultrafiltration goal. In 84 (65\%) PIRRT procedures, in which patients were initially under vasopressor therapy with norepinephrine, there was a median increase in norepinephrine dose of $+0.031 \mathrm{mcg} / \mathrm{kg} / \mathrm{min}$ during PIRRT (IQR 0.001 to +0.072 ), and $8(6 \%)$ patients required norepinephrine infusion during the PIRRT session.

Intradialytic hypotensive events occurred in 56 (43\%) procedures. Intradialytic hypotensive episodes were identified promptly in the majority of episodes and treated with 2 maneuvers: increasing norepinephrine dose
Table 1. Clinical characteristics of patients on the day of PIRRT initiation

Subjects' characteristics at PIRRT session onset $(n=21)$

Serum Cr, mg/dL

Blood urea nitrogen, $\mathrm{mg} / \mathrm{dL}$

Urine output, $\mathrm{mL} / \mathrm{d}$

Anuric patients (<100 mL/d), $n(\%)$

$\mathrm{PaO}_{2} / \mathrm{FiO}_{2}$

Hemoglobin, g/dL

WBC, $10^{3} / \mu \mathrm{L}$

Lymphocytes, $10^{3} / \mu \mathrm{L}$

Sodium, mEq/L

Potassium, mEq/L

C-reactive protein, $\mathrm{mg} / \mathrm{L}$

D-dimer, ng/mL

Ferritin, ng/mL

$4.6(4.2-5.8)$

$55(40-67)$

$189(45-390)$

$7(33)$

$123(102-151)$

$10.6(11.2-12.7)$

$11.7(8.0-13.6)$

$0.45(0.39-0.66)$

$139(137-142)$

$4.6(4.3-5.0)$

$35(19-61)$

$3,076(2,019-5,662)$

$1,038(513-1,350)$

Arterial blood gas

$\mathrm{pH}$

$7.22(7.19-7.28)$

$\mathrm{pCO}_{2}, \mathrm{~mm} \mathrm{Hg}$

$\mathrm{HCO}_{3}, \mathrm{mEq} / \mathrm{L}$

Arterial lactate, $\mathrm{mg} / \mathrm{dL}$

$55.1(46.4-63.0)$

$23(22.1-26.2)$

$1.3(1.8-2.3)$

Ventilator parameters

Plateau pressure, $\mathrm{cm} / \mathrm{H}_{2} \mathrm{O} \quad 27$ (23-29)

Static compliance, $\mathrm{cm} / \mathrm{H}_{2} \mathrm{O} \quad 34(27-37)$

PEEP, $\mathrm{cm} / \mathrm{H}_{2} \mathrm{O}$

$14(14-16)$

Prone positioning, $n(\%)$

Vasopressor use, $n(\%)$

$4(19)$

$21(100)$

Vasopressor dose, $\mu \mathrm{g} / \mathrm{kg} / \mathrm{min}$

$0.08(0.1-0.12)$

PIRRT, prolonged intermittent renal replacement therapy; PEEP, positive end-expiratory pressure. 
Table 2. Characteristics of the PIRRT sessions

\begin{tabular}{lc}
\hline Characteristic & \\
\hline Number of PIRRT sessions, $n$ & 130 \\
Number of PIRRT sessions per patient, $n$ & $5(4-9)$ \\
Treatment time, $\mathrm{h}$ [IQR] & $6(6-8)$ \\
Treatments that achieved full prescription time and ultrafiltrate goal, $n(\%)$ & $102(77)$ \\
Total duration of dialyzate effluent exposure, $\mathrm{h}$ [IQR] & $4(4-4)$ \\
Weight-adjusted ultrafiltration rate initially prescribed, mL/kg/h & $3.8(2.8-4.9)$ \\
Total fluid removal per session, L & $2.5(2.0-2.5)$ \\
Fluid removal per hour, mL/h & $313(125-416)$ \\
Dialyzer blood flow rate, mL/min & $200(200-250)$ \\
Dialyzate blood flow rate, mL/kg & $300(300-500)$ \\
Vasopressor therapy before PIRRT initiation, $n(\%)$ & $84(65)$ \\
Vasopressor therapy after PIRRT initiation, $n(\%)$ & $8(6)$ \\
Initial NOR dose, $\mu g / \mathrm{kg} / \mathrm{min}$ & $0.06(0.03-0.14)$ \\
Final NOR dose, $\mu \mathrm{g} / \mathrm{kg} / \mathrm{min}$ & $0.09(0.06-0.16)$ \\
Initial HR, bpm & $96(77-111)$ \\
Final HR, bpm & $101(84-110)$ \\
Initial SBP, mm/Hg & $116(105-130)$ \\
Final SBP, mm/Hg & $108(97-121)$ \\
Initial DBP, mm/Hg & $58(52-65)$ \\
Final DBP, mm/Hg & $58(54-62)$ \\
Treatments discontinued due to severe hypotensive episode, $n(\%)$ & $15(12)$ \\
Treatments in which clotting occurred at least once, $n(\%)$ & $17(13)$ \\
Treatments performed using heparin anticoagulation, $n(\%)$ & $116(89)$ \\
Mean heparin infusion rate per hour, U/h & $500(500-500)$ \\
Enoxaparin prescription, $n$ (\%) & $68(52)$
\end{tabular}

Pertreatment analysis. Bpm, beats per minute; PEEP, positive end-expiratory pressure; SBP, systolic blood pressure; DBP, diastolic blood pressure; HR, heart rate; NOR, norepinephrine; PIRRT, prolonged intermittent renal replacement therapy; IQR, interquartile range.

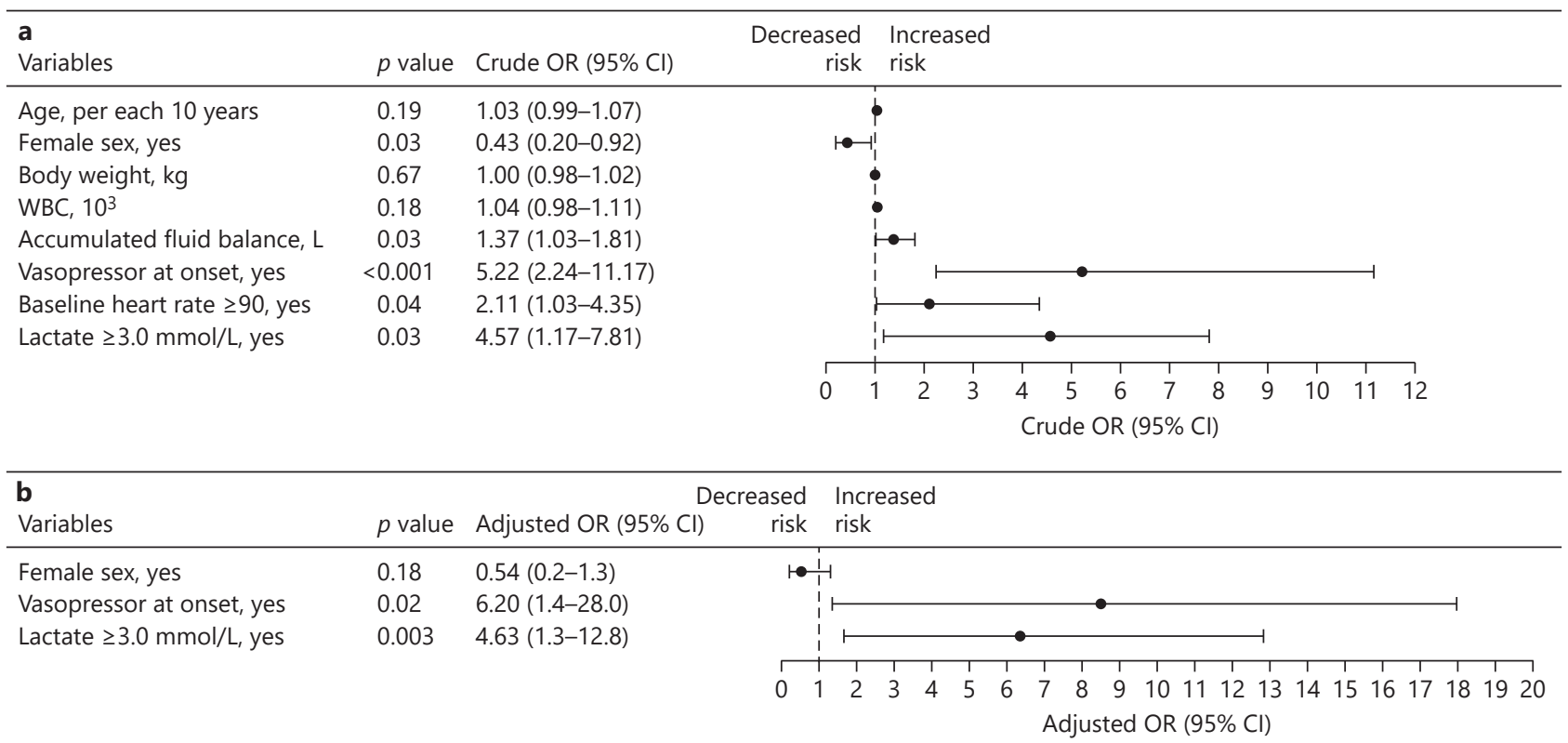

Fig. 2. Forest plot of risk factors associated with at least one intradialytic hypotensive episode in the univariate model (a) and retained in the multivariate model (b). +No-vasopressor at PIRRT session onset was the reference. 
Table 3. Characteristics of PIRRT procedures according to the occurrence of intradialytic hypotensive events

\begin{tabular}{|c|c|c|c|}
\hline Characteristic & $\begin{array}{l}\text { Hypotension during } \\
\text { PIRRT session }(n=56)\end{array}$ & $\begin{array}{l}\text { No hypotension during } \\
\text { PIRRT session }(n=74)\end{array}$ & $p$ value \\
\hline Total time of PIRRT, $\mathrm{h}$ & $7.5(6-8)$ & $6(5-8)$ & 0.002 \\
\hline Total duration of dialyzate effluent exposure, $\mathrm{h}$ & $4(4-4)$ & $4(4-4)$ & 1.00 \\
\hline Blood flow rate, $\mathrm{mL} / \mathrm{min}$ & $200(200-300)$ & $200(200-200)$ & 0.15 \\
\hline Dialyzate flow rate, $\mathrm{mL} / \mathrm{kg}$ & $300(300-500)$ & $300(300-300)$ & 0.081 \\
\hline $\mathrm{Hb}, \mathrm{g} / \mathrm{dL}$ & $10.3 \pm 2.2$ & $10.3 \pm 2.9$ & 0.91 \\
\hline $\mathrm{WBC}, 10^{3} / \mu \mathrm{L}$ & $13.6(10.3-16.9)$ & $11.5(8.8-14.5)$ & 0.022 \\
\hline $\mathrm{Na}, \mathrm{mEq} / \mathrm{L}$ & $135(133-138)$ & $135(134-138)$ & 0.42 \\
\hline $\mathrm{K}, \mathrm{mg} / \mathrm{dL}$ & $4.4 \pm 0.7$ & $4.3 \pm 0.7$ & 0.92 \\
\hline Ferritin, ng/mL & $520(330-1,342)$ & $953(519-1,336)$ & 0.086 \\
\hline Lactate, $\mathrm{mg} / \mathrm{dL}$ & $2.2(1.7-2.9)$ & $1.8(1.3-2.4)$ & 0.071 \\
\hline Lactate $\geq 3.0 \mathrm{mg} / \mathrm{dL}, n$ & $9(21 \%)$ & $3(4 \%)$ & 0.029 \\
\hline D-dimer, ng/mL & $3,906(2,126-6,362)$ & $4,294(, 2624-8,424)$ & 0.28 \\
\hline Prone position, $n$ & $11(19)$ & $18(24)$ & 0.52 \\
\hline $\mathrm{PEEP}, \mathrm{mL} / \mathrm{cm} / \mathrm{H}_{2} \mathrm{O}$ & $12(8-14)$ & $12(8-14)$ & 0.99 \\
\hline Static compliance, $\mathrm{mL} / \mathrm{cm} / \mathrm{H}_{2} \mathrm{O}$ & $35(29-44)$ & $32(25-43)$ & 0.29 \\
\hline $\mathrm{pH}$ & $7.25 \pm 0.07$ & $7.29 \pm 0.09$ & 0.10 \\
\hline $\mathrm{HCO}_{3}$ & $22.1 \pm 3.1$ & $23.1 \pm 3.5$ & 0.10 \\
\hline $\mathrm{pCO}_{2}$ & $49(40-57)$ & $50(40-58)$ & 0.92 \\
\hline Accumulated net fluid balance, $\mathrm{mL}$ & $96 \pm 1,655$ & $-447 \pm 1,025$ & 0.025 \\
\hline Total UF goal, L & $2.5(2-3.0)$ & $2.0(2.0-2.5)$ & 0.26 \\
\hline Initial UF rate per hour prescribed, $\mathrm{mL} / \mathrm{h}$ & $317 \pm 131$ & $326 \pm 162$ & 0.35 \\
\hline Initial UF rate adjusted weight, $\mathrm{mL} / \mathrm{kg} / \mathrm{h}$ & $3.8 \pm 1.5$ & $3.9 \pm 2.2$ & 0.85 \\
\hline UF rate per hour accomplished, $\mathrm{mL} / \mathrm{h}$ & $293 \pm 134$ & $368 \pm 139$ & 0.004 \\
\hline UF rate-adjusted weight accomplished, $\mathrm{mL} / \mathrm{kg} / \mathrm{h}$ & $3.6 \pm 1.4$ & $3.7 \pm 1.6$ & 0.72 \\
\hline Vasopressor therapy, $n(\%)$ & $47(56)$ & $37(44)$ & 0.001 \\
\hline Initial NOR, $\mu \mathrm{g} / \mathrm{kg} / \mathrm{min}$ & $0.05(0.01-0.12)$ & $0.05(0.03-0.14)$ & 0.31 \\
\hline Final NOR, $\mu \mathrm{g} / \mathrm{kg} / \mathrm{min}$ & $0.11(0.07-0.20)$ & $0.06(0.03-0.15)$ & 0.017 \\
\hline Change in NOR dose, $\mu \mathrm{g} / \mathrm{kg} / \mathrm{min}$ & $+0.07(0.04-0.09)$ & $0(0-0.01)$ & $<0.001$ \\
\hline Temperature, ${ }^{\circ} \mathrm{C}$ & $37(36.8-38.6)$ & $37(36.5-37.6)$ & 0.35 \\
\hline Initial SBP, mm Hg & $120 \pm 25$ & $119 \pm 18$ & 0.64 \\
\hline Initial DBP, mm Hg & $60 \pm 11$ & $59 \pm 11$ & 0.42 \\
\hline Final SBP, $\mathrm{mm} \mathrm{Hg}$ & $110 \pm 22$ & $111 \pm 19$ & 0.55 \\
\hline Final DBP, mm Hg & $62 \pm 17$ & $59 \pm 11$ & 0.22 \\
\hline Initial HR, bpm & $97(79-113)$ & $90(65-105)$ & 0.016 \\
\hline Final HR, bpm & $105(91-127)$ & $95(77-106)$ & 0.019 \\
\hline
\end{tabular}

Data are presented as mean \pm standard deviation, median (interquartile range), or $n(\%)$. All variables are shown at the PIRRT session onset. NOR, norepinephrine; SBP, systolic blood pressure; DBP, diastolic blood pressure; HR, heart rate; PIRRT, prolonged intermittent renal replacement therapy; PEEP, positive end-expiratory pressure.

$(54 / 56,96 \%)$ and reducing fluid removal rate by decreasing total ultrafiltrate $(10 / 56,18 \%)$ or by increasing session duration $(46 / 56,82 \%)$. Fifteen (12\%) PIRRT treatments were discontinued before reaching the ultrafiltration goal due to severe intradialytic hypotension. Other causes of PIRRT discontinuation were recurrent dialysis system clotting, arrhythmia, and refractory hypoxemia in 5, 1, and 1 procedure, respectively. Two patients were switched to CRRT due to severe hypotension after 2 consecutive PIRRT treatments failed on subsequent days.
The median (IQR) proportion of intradialytic hypotensive events per each patient (the frequency of hypotensive events divided by the total number of PIRRT treatments per patient) was 0.4 (0.1-0.7). Men had a higher proportion of intradialytic hypotensive events than women $(0.64[0.30-0.73]$ vs. 0.40 [0.11-0.60], $p: 0.032)$. The characteristics of PIRRT sessions complicated by intradialytic hypotensive events compared to those without hypotensive episodes are displayed in Table 3. As it is shown, PIRRT sessions complicated by intradialytic hy- 
Table 4. Suggestion for PIRRT in COVID-19 patients with ARDS

\begin{tabular}{lll}
\hline PIRRT-related factor & Suggestion & Rationale according to our observational data \\
\hline $\begin{array}{ll}\text { "Early" placement of } \\
\text { vascular access }\end{array}$ & $\begin{array}{l}\text { In high-risk patients for RRT, } \\
\text { place a CVC before adopting } \\
\text { prone position }\end{array}$ & $\begin{array}{l}\text { During the first weeks of pandemic, urgent CVC placements were complicated by the prone } \\
\text { position of patients with severe ARDS in our cohort }(n=4) \text {. Turning to supine position for } \\
\text { timely CVC insertion was not possible in 2 patients; fortunately, PIRRT could be delayed } \\
\text { without severe complications. Although safe ultrasound-guided CVC placement in prone } \\
\text { position has been described [9], we decided to adopt an "early" placement of CVC before } \\
\text { adopting prone position in those subjects with mechanical ventilation and high risk for RRT }\end{array}$ \\
\hline
\end{tabular}

Timing for initiation Reduce net positive fluid balance In our ICU, a strategy of conservative fluid management has been employed in the majority of of PIRRT until a complete resuscitation fluid is confirmed. Consider high insensible fluid losses related to persistent fever. Bedside ultrasound-guided volume could be useful to assess fluid status patients according to the recommendations for ARDS. In this regard, the median cumulative fluid balance from admission to PIRRT initiation was 3.56 (range 1.8-6.7) L. Nevertheless, cumulative fluid balance charts were frequently inaccurate compared to clinical and ultrasound assessment given high insensible fluid losses related to persistent fever. For example, 12 nonPIRRT patients with stage 3 AKI and anuria were frankly depleted by ultrasound-guided volume assessment and responded to fluid repletion. We tried to delay PIRRT initiation until a complete resuscitation was documented, including ultrasound volume markers when possible, allowing time to assess the response to fluids and furosemide test

Limit COVID-19 If possible, limit hemodialysis exposure staff exposure

Aiming to limit exposure among hemodialysis staff, and to limit the use of personal protective equipment, patients with COVID-19 and AKI who required PIRRT were placed close together in the ICU when possible. Thus, one nurse simultaneously delivered dialysis to more than one patient in adjoining beds for a maximum period of $4-6 \mathrm{~h}$; when the PIRRT is longer than that, a second nurse would come to replace the first one

\begin{tabular}{ll}
\hline $\begin{array}{l}\text { Prothrombotic } \\
\text { complications }\end{array}$ & Use of systemic anticoagulation \\
and regional anticoagulation
\end{tabular}

Circuit thrombotic events were frequent during the first 2 weeks of the COVID-19 outbreak. complications and regional anticoagulation The institutional protocol adopted the use of systemic anticoagulation (enoxaparin SC $0.5 \mathrm{mg}$ / $\mathrm{kg} / \mathrm{d}$ ), and during PIRRT, we added regional anticoagulation using unfractionated heparin $(500 \mathrm{U} / \mathrm{h})$. Anti-factor Xa activity was measured every 4 days with a target anti-Xa activity of $0.6-1.0$

Severe acidemia Increased dialyzate base yet avoid sudden $\mathrm{pH}$ normalization

\begin{abstract}
Permissive hypercapnia caused by low tidal volume ventilation $(6 \mathrm{~mL} / \mathrm{kg}$ of predicted body weight) was associated to arterial $\mathrm{pH}$ below 7.25 before PIRRT initiation in the majority of patients (11/14), despite prescription of large volumes of bicarbonate in all patients (range $100-250 \mathrm{mEq} / \mathrm{d}[11])$. In general, we increased dialyzate base $+4 \mathrm{mEq} / \mathrm{L}(32 \mathrm{mEq} / \mathrm{L})$ and prescribed dialyzate flows of $300 \mathrm{~mL} / \mathrm{min}$ for no more than $4 \mathrm{~h}$, intended to achieve a $\mathrm{pH}$ > 7.25 , avoiding sudden $\mathrm{pH}$ normalization
\end{abstract}

PIRRT, prolonged intermittent renal replacement therapy; CVC, central venous catheter; RRT, renal replacement therapy; ARDS, acute respiratory distress syndrome; AKI, acute kidney injury; ICU, intensive care unit.

potensive events had longer duration, less fluid removal per hour, and a higher increase in vasopressor dose, and occurred more frequently in male patients with higher WBC counts, heart rate frequencies, and arterial lactate concentrations. We performed a multivariate analysis of variables associated with intradialytic hypotensive events using the variables shown in Figure 2. Vasopressor at PIRRT session onset (OR 6.2, 95\% CI 1.4-28.0, p:0.02) and a pre-PIRRT lactate $\geq 3.0 \mathrm{mmol} / \mathrm{L}$ (OR $4.63,95 \%$ CI $1.3-12.8, p: 0.003)$ were independently and significantly associated with the risk of hypotension during PIRRT.

Circuit thrombotic events were frequent during the first 2 weeks of the COVID-19 outbreak. In the first 30 PIRRTs, 7/30 (23\%) clotting events occurred at least once. Two central venous catheters were removed due to irreversible occlusion of the lumen by thrombus. Nurse and laboratory staff shortages prevented the use of total coagulation with unfractionated heparin since adequate monitoring of coagulation times was not possible. Therefore, the institutional protocol adopted the use of systemic anticoagulation (enoxaparin SC $0.5 \mathrm{mg} / \mathrm{kg} /$ day), and during PIRRT, we added regional anticoagulation using unfractionated heparin $(500 \mathrm{U} / \mathrm{h})$. Anti-factor Xa activity was measured every 4 days $3-4 \mathrm{~h}$ after enoxaparin application with a target anti-Xa activity of $0.6-1.0 \mathrm{UI} / \mathrm{mL}$ [10]. After these maneuvers, the proportion of treatments in which clotting occurred at least once decreased from 23 to $10 \%(10 / 100, p<0.001)$. After double anticoagulation, 2 cases presented minor bleeding which resolved after discontinuation of enoxaparin. We introduced other changes in our PIRRT protocol during the COVID-19 outbreak to maximize resources and to address the spe- 
cific needs of COVID-19 patients, which are shown in Table 4.

During follow-up, 11 patients (52\%) recovered from AKI and respiratory failure, 9 (43\%) patients died, and 1 (5\%) patient is still hospitalized at the time of writing this report, yet he has recovered renal function and, subsequently, PIRRT has already been discontinued. In those patients who recovered renal function $(12 / 21)$, the median number of PIRRT sessions was 5 (IQR 4-10) and the median time of follow-up was 28 (14-62) days. None of the deaths were directly or indirectly related to adverse events during the PIRRT session.

\section{Discussion/Conclusion}

In the last 14 weeks, our ICUs unexpectedly faced multiple challenges for delivering timely RRT in multiple extremely ill patients, including shortages of nursing staff, vital equipment, and hemodialysis machines. Our main findings are that (1) PIRRT sessions were feasible in almost $85 \%$ of procedures, (2) intradialytic hypotensive episodes were a frequent event in these patients and were mainly related to inflammatory status and organ hypoperfusion assessed by arterial lactate and vasopressor use, (3) the majority of the hypotensive episodes were identified and successfully treated with reduction of planned fluid removal during PIRRT session and increasing session duration, and (4) systemic anticoagulation with lowmolecular-weight heparin and regional anticoagulation of the PIRRT system reduced the high incidence of the circuit and access thrombosis observed in ICU patients with COVID-19 infection.

Contrary to the initial reports of a very low incidence of AKI associated to SARS-CoV-2 [12], recent reviews estimated an AKI occurrence between 8.3 and $23 \%$ and a frequency of RRT requirement of $14.6-58.3 \%$ in critically ill COVID-19 patients who developed AKI $[13,14]$. In our cohort, we found a high AKI occurrence of $60.7 \%$ during the ICU stay, and among them, severe AKI (stage 3) occurred in almost one-third of cases. These discrepancies between our results and others could be explained by the strict application of KDIGO criteria, a high prevalence of diabetes and hypertension, the severity of ARDS, and the longer stay in the ICU. Nevertheless, the proportion of critically ill COVID-19 cases needing RRT in all patients admitted to the ICU was $<10 \%$, which is lower than previous projections of $25 \%$ suggested by other authors [15].

Survival data of COVID-19 patients who needed RTT are incomplete in the literature so far published. Yang et al. [16] and Zhou et al. [17] showed a mortality in severely ill patients treated with CRRT of $89 \%(n=8 / 9)$ and $100 \%(n=10 / 10)$, respectively. In a recent meta-analysis, CRRT initiation was a poor prognosis factor in severe COVID-19, given that $15.6 \%$ of non-survivors of COVID-19 received CRRT compared to $0.4 \%$ of survivors [18]. In our center, we observed a mortality of $<50 \%$ in patients treated with PIRRT. A hopeful observation is that more than half of the patients treated with PIRRT survived, recovered kidney function, and were discharged within 28 days after RRT initiation. It is conceivable that our survival outcomes are most likely the result of many factors, including ICU team's competition and performance, but our data provide reassurance of continuing to use PIRRT in critically ill COVID-19 patients.

The frequency of intradialytic hypotensive episodes observed was within the range of other non-COVID-19 cohorts in the ICU treated with PIRRT, which is between 17 and 57\% [19-23]. The requirement of any dose of vasopressor or pre-PIRRT elevated arterial lactate, both of which are related to the severity of COVID-19 infection, was strongly related to hypotensive episodes. Despite all the adopted measures to avoid intradialytic hypotension in this cohort, there were no differences in PIRRT prescription between those PIRRT procedures which presented hypotensive episodes and those without this complication. Extending the total time of PIRRT procedures or decreasing the ultrafiltrate rate to $<400 \mathrm{~mL} / \mathrm{h}$ in hemodynamically unstable patients was not effective in decreasing the risk of hypotensive events, as it has been described in other series [24]. Although CRRT modality is presumed to have the least impact on hemodynamic stability, the superiority of CRRT over PIRRT to prevent hypotensive episodes and other outcomes such as renal recovery and mortality is controversial $[25,26]$. The advantage of performing several dialysis procedures with short nursery staff and at low cost constitutes a very important advantage of PIRRT over CRRT in our center. For example, at our hospital, the CRRT supplies cost is approximately 17 fold higher than that of the PIRRT supplies (USD 455 vs. USD 27, respectively). Nevertheless, we believe that our COVID-19 patients with multiorgan failure demand better modalities of RRT that achieve hemodynamic stability, decrease adverse events during treatments, and provide alternative ways to improve clinical outcomes, for example, the removal of cytokines during "the cytokine storm period" related to SARS-CoV-2 infection [5].

Even peritoneal dialysis is a common choice of replacement therapy in Mexico, and few authors have suggested this modality as a feasible option during the CO- 
VID-19 outbreak [27, 28], yet it was not achievable due to the need for prone positioning in most of the COVID-19 patients with ARDS. Other factors that may have made it not reasonable to adopt this strategy at our center would have been an insufficient number of trained staff, exposure risk associated with frequent fluid exchanges, and the difficulties for timely peritoneal catheter placement.

Our study has limitations. The observational design of this study limits its generalizability, the lack of significant differences in many clinical characteristics associated with adverse events could be due to the small number of patients, and our suggestions must be validated in prospective and multicenter trials to confirm our hypothesis. So far, we did not find studies about PIRRT feasibility in critically ill COVID-19 patients with ARDS and severe AKI.

In conclusion, PIRRT may represent an acceptable alternative for other centers with circumstances similar to our managing COVID-19 patients with ARDS and severe AKI. In these extraordinary circumstances, PIRRT therapy was feasible in the majority of our patients who exhibited an exuberant inflammatory response, severe hemodynamic instability, and hypercoagulability.

\section{Acknowledgements}

The authors greatly appreciate all of the hospital staff for their efforts in treating patients and thank all patients involved in this study.

\section{Statement of Ethics}

Patients' informed consent was obtained, and the protocol was approved by the institutional review board (number protocol NMM-3325-20-20-1).

\section{Conflict of Interest Statement}

All authors declare that they have no competing interests, no support from any organization for the submitted work, and no financial relationship with any organizations that might have an interest in the submitted work. No pharmaceutical or biotechnology company, foundation, or any other source participated in the design, monitoring, data collection, and analysis. Dr. Juan Carlos Ramírez Sandoval had the following relationship with companies: Takeda Mexico (speakers' bureau), Roche Mexico (speakers' bureau), Amgen Mexico (speakers' bureau), Valdecasas Mexico (research funding), and Mercurio S.A. de C.V (advisory). Dr. Mauricio Arvizu Hernández had received funding for academic meetings and honoraria as speakers' bureau from Medtronic. Dr. Ricardo Correa Rotter is a member of the Review Board of Blood Purification, has received honoraria from AbbVie, AstraZeneca, GlaxoSmithKline, and Boehringer Ingelheim, and has lectured for Amgen, Takeda, AstraZeneca, and Roche. Dr. Olynka Vega Vega has received honoraria from Amgen, Baxter, and Boehringer Ingelheim and had received a grant for research funding from Baxter Mexico. Dr. Jorge E. Gaytán Arocha, Pedro Xolalpa Chávez, Juan Manuel Mejía Vilet, Eduardo Rivero Sigarroa, and Carlos Torruco Sotelo have no conflicts of interest to declare.

\section{Funding Sources}

The authors received no specific funding for this work.

\section{Author Contributions}

All authors conceived and designed the study. All authors performed the inclusion of subjects and prospective follow-up. J.C.R.S., J.E.G.A., P.X.C., J.M.M.V., M.A.H., E.R.S., C.T.S., R.C.R., and O.V.V. analyzed the data and reviewed the results. J.C.R.S., J.M.M.V., R.C.R., and O.V.V. wrote the manuscript. All authors revised the manuscript.

\section{References}

1 Manns B, Doig CJ, Lee H, Dean S, Tonelli M, Johnson $\mathrm{D}$, et al. Cost of acute renal failure requiring dialysis in the intensive care unit: clinical and resource implications of renal recovery. Crit Care Med. 2003;31(2):449-55.

2 Tolwani AJ, Wheeler TS, Wille KM. Sustained low-efficiency dialysis. Contrib Nephrol. 2007;156:320-4.

3 Ronco C, Bagshaw SM, Bellomo R, Clark WR, Husain-Syed F, Kellum JA, et al. Extracorporeal blood purification and organ support in the critically ill patient during COVID-19 pandemic: expert review and recommendation. Blood Purif. 2020:1-11.
4 Maoujoud O, Asserraji M, Belarbi M, Zemraoui N. Coronavirus disease 19 pandemic and extracorporeal therapies in intensive care: "bis peccare in bello non licet". Blood Purif. 2020:1-2.

5 Al Shareef K, Bakouri M. Cytokine blood filtration responses in COVID-19. Blood Purif. 2020:1-9.

6 ARDS Definition Task Force; Ranieri VM, Ranieri VM, Rubenfeld GD, Thompson BT, Ferguson ND, et al. Acute respiratory distress syndrome: the Berlin definition. JAMA. 2012; 307(23):2526-33.
7 Kooman J, Basci A, Pizzarelli F, Canaud B, Haage P, Fouque D, et al. EBPG guideline on haemodynamic instability. Nephrol Dial Transplantation. 2007;22(Suppl 2):ii22-44.

8 Kidney Disease: Improving Global Outcomes (KDIGO) Acute Kidney Injury Work Group. KDIGO clinical practice guideline for acute kidney injury. Kidney Int Suppl. 2012;2:1138.

9 Lazzari A, Bini R, Bertucci R, Coletti A, Voghera P. Ultrasound-guided intravenous catheter insertion in a prone patient using a $\mathrm{T}$ probe in emergency setting. J Vasc Access. 2017;18(6):e95-7. 
10 Klok FA, Kruip MJHA, van der Meer NJM, Arbous MS, Gommers DAMPJ, Kant KM, et al. Incidence of thrombotic complications in critically ill ICU patients with COVID-19. Thromb Res. 2020;191:145-7.

11 Jaber S, Paugam C, Futier E, Lefrant JY, Lasocki $\mathrm{S}$, Lescot T, et al. Sodium bicarbonate therapy for patients with severe metabolic acidaemia in the intensive care unit (BICARICU): a multicentre, open-label, randomised controlled, phase 3 trial. Lancet. 2018; 392(10141):31-40.

12 Wang L, Li X, Chen H, Yan S, Li D, Li Y, et al. Coronavirus disease 19 infection does not result in acute kidney injury: an analysis of 116 hospitalized patients from Wuhan, China. Am J Nephrol. 2020;51(5):343-8.

13 Gabarre P, Dumas G, Dupont T, Darmon M, Azoulay E, Zafrani L. Acute kidney injury in critically ill patients with COVID-19. Intensive Care Med. 2020;46(7):1339-48.

14 Yang X, Jin Y, Li R, Zhang Z, Sun R, Chen D. Prevalence and impact of acute renal impairment on COVID-19: a systematic review and meta-analysis. Crit Care. 2020;24(1):356.

15 Neyra JA, Connor MJ Jr, Tolwani A. Preparedness of kidney replacement therapy in the critically ill during COVID-19 surge. Kidney Int Rep. 2020;5(7):961-4.

16 Yang X, Yu Y, Xu J, Shu H, Xia J, Liu H, et al. Clinical course and outcomes of critically ill patients with SARS-CoV-2 pneumonia in Wuhan, China: a single-centered, retrospective, observational study. Lancet Respir Med. 2020;8(5):475-81.
17 Zhou F, Yu T, Du R, Fan G, Liu Y, Liu Z, et al. Clinical course and risk factors for mortality of adult inpatients with COVID-19 in Wuhan, China: a retrospective cohort study. Lancet. 2020;395(10229):1054-62.

18 Yang X, Jin Y, Li R, Zhang Z, Sun R, Chen D. Prevalence and impact of acute renal impairment on COVID-19: a systematic review and meta-analysis. Crit Care. 2020;24(1):356.

19 Schortgen F, Soubrier N, Delclaux C, Thuong M, Girou E, Brun-Buisson C, et al. Hemodynamic tolerance of intermittent hemodialysis in critically ill patients:usefulness of practice guidelines. Am J Respir Crit Care Med. 2000; 162:197-202.

20 Vinsonneau C, Camus C, Combes A, Costa de Beauregard MA, Klouche K, Boulain T, et al. Continuous venovenous haemodiafiltration versus intermittent haemodialysis for acute renal failure in patients with multiple-organ dysfunction syndrome: a multicentre randomised trial. Lancet. 2006;368(9533):37985.

21 Bitker L, Bayle F, Yonis H, Gobert F, Leray V, Taponnier R, et al. Prevalence and risk factors of hypotension associated with preload-dependence during intermittent hemodialysis in critically ill patients. Crit Care. 2016;20:44.

22 du Cheyron D, Lucidarme O, Terzi N, Charbonneau P. Blood volume- and blood temperature-controlled hemodialysis in critically ill patients: a 6-month, case-matched, open-label study. Blood Purif. 2010;29(3):245-51.
23 du Cheyron D, Terzi N, Seguin A, Valette X, Prevost F, Ramakers M, et al. Use of online blood volume and blood temperature monitoring during haemodialysis in critically ill patients with acute kidney injury: a single centre randomized controlled trial. Nephrol Dial Transplant. 2013;28:430-7.

24 Albino BB, Balbi AL, Abrão JM, Ponce D. Dialysis complications in acute kidney injury patients treated with prolonged intermittent renal replacement therapy sessions lasting 10 versus $6 \mathrm{~h}$ : results of a randomized clinical trial. Artif Organs. 2015;39(5):423-31.

25 Akhoundi A, Singh B, Vela M, Chaudhary S, Monaghan M, Wilson GA, et al. Incidence of adverse events during continuous renal replacement therapy. Blood Purif. 2015;39(4): 333-9.

26 Chen Y. Effect of renal replacement therapy modalities on renal recovery and mortality for acute kidney injury: A PRISMA-compliant systematic review and meta-analysis. Semin Dial. 2020;33(2):127-32.

27 Goldfarb DS, Benstein JA, Zhdanova O, Hammer E, Block CA, Caplin NJ, et al. Impending shortages of kidney replacement therapy for COVID-19 patients. Clin J Am Soc Nephrol. 2020;15(6):880-2.

28 Bittencourt Dias D, Mendes ML, Alves CA, Caramori JT, Ponce D. Peritoneal dialysis as an urgent-start option for incident patients on chronic renal replacement therapy: world experience and review of literature. Blood $\mathrm{Pu}$ rif. 2020:1-6. 